\title{
Peripheral Nerve Involvement in HTLV-I-Associated Myelopathy: Report of a Case
}

\author{
Kouji FUnAmOTO, Kuniyasu TAKADA*, Kazuhiko InOUE, Yoshihiro SAwADA, \\ Shigeru ARAGA and Kazuro TAKAHASHI
}

\begin{abstract}
A patient came to our clinic suffering from mild sensory loss, exaggeration in knee reflexes, bilateral extensor plantar responses and elevation of the antibody level to the human T-lymphotropic virus type I (HTLV-I). Electrophysiological and pathological studies showed axonal and demyelinating neuropathy. The condition appeared to be a slowly progressive myeloneuropathy. HTLV-I may play an important role in the pathogenesis of myeloneuropathy.
\end{abstract}

Key words: HTLV-I, Myelopathy, Neuropathy, HIV

The human T-lymphotropic virus type I (HTLVI) associated myelopathy (HAM) is characterized by gradual onset and a slowly progressive course, spastic paraparesis with pyramidal signs associated with mild sensory and sphincter disturbances (1). Recently, peripheral nerve involvements in HAM have been described $(2,3)$. This case is suggestive of HTLV-I-associated myeloneuropathy.

\section{CASE REPORT}

A 64-year-old woman from Yonago, in western Japan, had a 2-year history of gradual worsening of plantar hyperalgesia. On examination in November 1988, she showed exaggeration in knee reflexes with normal ankle reflexes. There were bilateral extensor plantar responses, plantar hypesthesia to pinprick and touch, and normal position sense. There was no muscle weakness or atrophy. Otherwise, she was neurologically normal.

Complete blood cell counts were normal; the erythrocyte sedimentation rate was $16 \mathrm{~mm}$ per 1 hour. C-reactive protein was negative. Tests for rheumatoid factor, systemic lupus erythematosus and syphilis were negative. The oral glucose tolerance test $(75 \mathrm{~g})$ showed a normal pattern. Serum vitamin $B_{1}$ and $B_{12}$ were normal. Serum immunoglobulins were within normal ranges, and there were no M-proteins found by immunoelectrophoresis. Anti-HTLV-I antibody was detected in the serum of the patient by the passive particle-agglutination (PA) test at a dilution of 1:10540. The cerebrospinal fluid (CSF) contained 3 lymphocytes per milliliter and $25 \mathrm{mg}$ protein/dl with $2.9 \mathrm{mg}$ IgG. Anti-HTLVI antibody in CSF was detected at a dilution of 1:64. Antibody to the human immunodeficiency virus (HIV) was found to be serologically negative by enzyme-linked immunosorbent assay. Cervical and lumbar X-rays showed mild spondylotic changes without narrowing of the spinal canal. Brain CT scans were normal. Cranial, cervical, thoracic and lumbar magnetic resonance imaging were not significant. The electrodiagnostic studies showed the following abnormalities (Table 1); reduction of sensory nerve action potential and conduction velocity in the right sural nerve; disappearance of sensory nerve action potential in the left sural nerve; and reduction of the amplitude of the $M$ responses in the bilateral peroneal nerves. Signs of mild

From Divisions of Neurology and *Neuropathology, Institute of Neurological Sciences,

Tottori University School of Medicine, Yonago

Received for publication May 15, 1989.

Reprint request to: Kouji Fumamoto, MD, Division of Neurology, Institute of Neurological Sciences,

Tottori University School of Medicine, Nishi-machi 86, Yonago 683, Japan 
Table 1. Nerve conduction values.

\begin{tabular}{lrr}
\hline & Right & Left \\
\hline Posterior tibial & & \\
Amplitude (mV) & 5.1 & 8.4 \\
Distal latency (msec) & 4.0 & 3.8 \\
Conduction velocity (m/sec) & 44.0 & 45.6 \\
F-wave latency (msec) & 44.8 & 44.0 \\
Peroneal & & \\
Amplitude (mV) & 0.1 & 0.5 \\
Distal latency (msec) & 5.7 & 5.1 \\
Conduction velocity (m/sec) & 49.7 & 49.2 \\
F-wave latency (msec) & 55.7 & 51.9 \\
Sural & & \\
SNAP ( (m) & 3.5 & $\mathrm{NR}$ \\
Distal latency (msec) & 2.7 & \\
Conduction velocity (m/sec) & 41.0 & \\
\hline
\end{tabular}

$\mathrm{SNAP}=$ sensory nerve action potential, $\mathrm{NR}=$ no response

neurogenic changes were found by needle electromyography (EMG) with a reduction in the number of motor unit potentials and mild high amplitude potentials in the tibial anterior and gastrocnemius muscles.

Biopsies of the left sural nerve and the peroneus brevis muscle were performed. The nerve specimens were prepared for light and electron microscopy. Teased nerve fibers were prepared for light microscopy only. The frozen muscle sections were stained with hematoxylin-eosin, modified Gomori's trichrome and a battery of histochemical stains. In Epon-embedded specimens, there were many nerve fibers with a thin myelin sheath suggesting remyelination (Fig. 1). No cellular infiltration was found in the endoneurium or around the epineurial blood vessels. The density of myelinated fiber was $8571 / \mathrm{mm}^{2}$ in endoneurial areas. This exhibited a shift to the left of the peak of large myelinated fibers, and mild reduction of the amplitude of this peak in the histogram of the myelinated fibers (Fig. 2). The unmyelinated fiber density was $31107 / \mathrm{mm}^{2}$, which was normal. Teased fiber studies revealed that $71.4 \%$ of the fibers were normal. $26.7 \%$ of the fibers showed segmental abnormalities of the myelin sheath such as: $2.9 \%$ with paranodal demyelination; $5.7 \%$ with segmental demyelination and remyelination; and $18.1 \%$ with segmental remyelination. $1.9 \%$ of the fibers exhibited axonal degeneration. In the

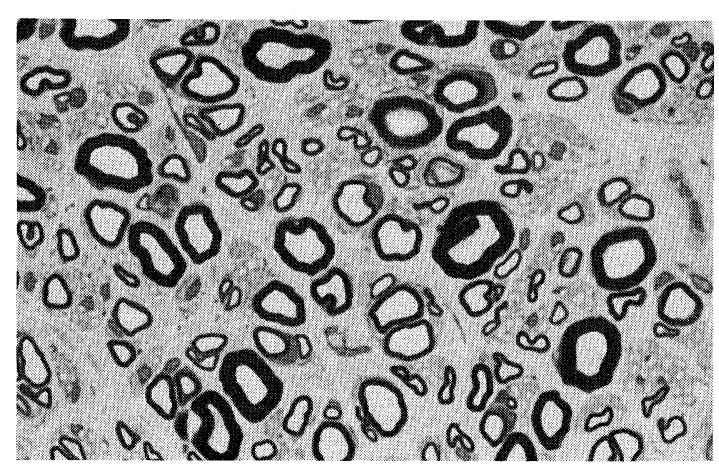

Fig. 1. Epon-embedded sections of the sural nerve. (Toluidine blue stain, $\times 600$ ).

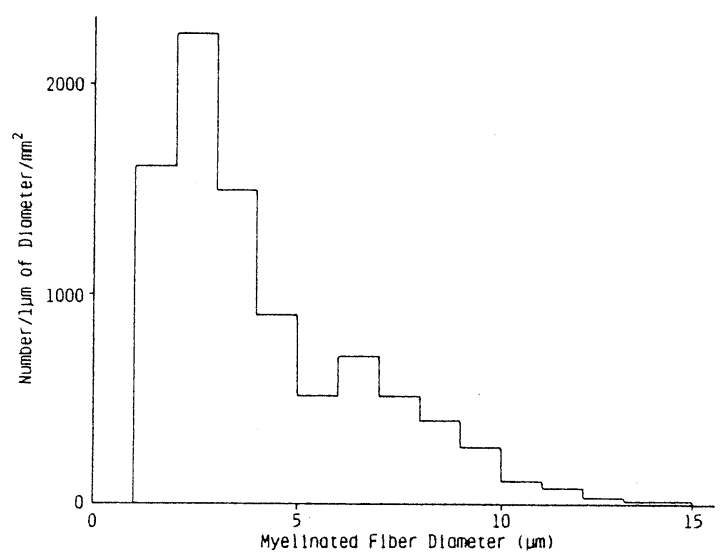

Fig. 2. The histogram of the myelinated fibers.

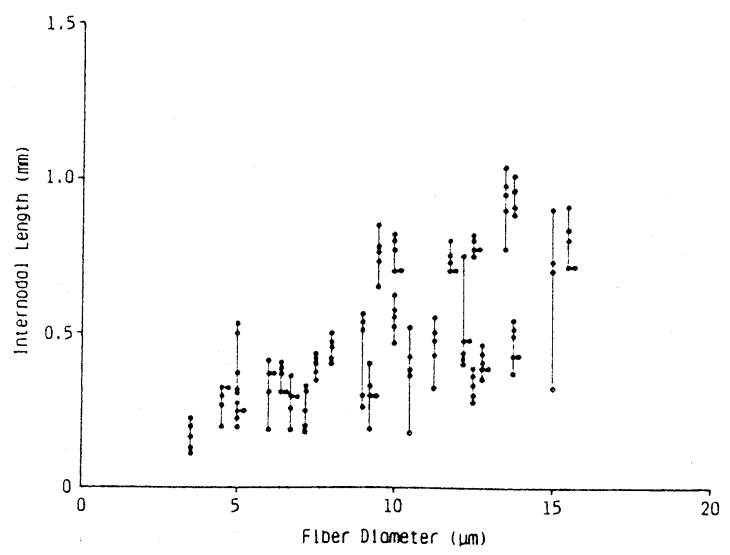

Fig. 3. The diagram of internodal length in relation to fiber diameter. 
diagram of internodal length in relation to fiber diameter (Fig. 3), many fibers exhibited increased variability of internodal length, suggesting remyelination after focal demyelination. In the frozen serial sections of the muscle specimens, some groups of small angulated fibers suggested denervation atrophy. There were no indications of endomysial or perimysial fibrosis and no cellular infiltration around the blood vessels.

Plasmapheresis for one day and pulse therapy with methylprednisolone of $1,000 \mathrm{mg} /$ day for 3 days were repeated twice within a 5-week period, but her symptoms were not improved.

\section{DISCUSSION}

Our patient had symmetrical plantar hypesthesia to pinprick and touch, exaggeration of knee reflexes and plantar extensor responses. The electrophysiological studies showed axonal and demyelinating neuropathy. The main histological findings were mild axonal loss of the large myelinated fibers, segmental demyelination and remyelination of the sural nerve and denervation atrophy of muscle fiber. Other causes of neuropathy, such as diabetes mellitus, paraproteinemia, vitamin deficiency and collagen diseases, were ruled out. The patient was diagnosed as HTLV-I-associated myeloneuropathy.

The pathological findings of HAM have been reported mainly concerning proliferation of capillaries, perivascular cuffing with lymphocytes and loss of myelin and axon in the lateral and anterior columns (4). Arimura et al (5) reported that peripheral nerve conduction studies in six patients with HAM were unremarkable, but the F-wave conduction velocities (FWCVs) of some patients were delayed and needle EMG studies showed neurogenic changes. They interpreted these findings as showing mild involvement of either the proximal portions of the peripheral motor pathway or the anterior horn cells of the spinal cord. Said et al (2) reported on a patient with HAM with a slowly progressive, mixed, demyelinating and axonal neuropathy of inflammatory origin. Also, cellular infiltrates predominated in the epineurium, especially around the blood vessels. Watanabe et al (3) reported on two patients with HAM showing numerous segmental demyelination and remyelination with globular formation. A mild decrease in the density of the myelinated fibers was noted. Although our patient showed slowly progressive myeloneuropathy similar to the above cases, neither cellular infiltration nor globular formation were found.

Besides involvement of the central nervous system, the peripheral nerves and the muscles have been extensively affected in patients with the acquired immunodeficiency syndrome (AIDS) or AIDS-related complex (ARC) (6-8). In such patients, several forms of peripheral neuropathy including acute or chronic inflammatory demyelinating polyneuropathy, distal symmetrical polyneuropathy, polyradiculopathy and mononeuropathy multiplex have been described $(6,7)$. Our patient indicated clinical findings of distal symmetrical polyneuropathy similar to AIDS or ARC.

HTLV-I and HIV belong to the same family of retroviruses. HTLV-I may be pathogenic in patients with myeloneuropathy.

\section{REFERENCES}

1) Osame M, Usuku K, Izumo S, et al: HTLV-I associated myelopathy, a new clinical entity. Lancet i: 1031, 1986.

2) Said G, Goulon-Goeau C, Lacroix $C$, et al: Inflammatory lesions of peripheral nerve in a patient with human T-lymphotropic virus type I-associated myelopathy. Ann Neurol 24: 275, 1988.

3) Watanabe M, Mano K, Watanabe, H, et al: Sural nerve biopsy of HTLV-I associated myelopathy. Neurol Med (Tokyo) 29: 504, 1988.

4) Akizuki S, Nakazato O, Higuchi $Y$, et al: Necropsy findings in HTLV-I associated myelopathy. Lancet i: 156, 1987.

5) Arimura $K$, Rosales $R$, Osame $M$, et al: Clinical electrophysiologic studies of HTLV-I-associated myelopathy. Arch Neurol 44: 609, 1987.

6) Snider WD, Simpson DM, Nielsen S, et al: Neurological complications of acquired immune deficiency syndrome: Analysis of 50 patients. Ann Neurol 14: 403, 1983.

7) Parry GJ: Peripheral neuropathies associated with human immunodeficiency virus infection. Ann Neurol 23 (suppl): S49, 1988

8) Simpson DM, Bender AN: Human immunodeficiency virus-associated myopathy: analysis of 11 patients. Ann Neurol 24: 79, 1988. 